OPEN ACCESS

Edited by:

Yi Zhao,

Beijing University of Chinese

Medicine, China

Reviewed by:

Jie Sun,

Harbin Medical University, China

Fei He,

University of Missouri, United States

Meng Zhou,

Wenzhou Medical University, China

*Correspondence:

Haining Cui

cuihn@jlu.edu.cn

Ying Xu

xyn@uga.edu

Specialty section:

This article was submitted to

Bioinformatics and Computational

Biology,

a section of the journal

Frontiers in Genetics

Received: 24 February 2020

Accepted: 20 April 2020

Published: 19 May 2020

Citation:

Liu L, Cui H and Xu Y (2020)

Quantitative Estimation of Oxidative Stress in Cancer Tissue Cells Through

Gene Expression Data Analyses.

Front. Genet. 11:494.

doi: 10.3389/fgene.2020.00494

\section{Quantitative Estimation of Oxidative Stress in Cancer Tissue Cells Through Gene Expression Data Analyses}

\author{
Liyang Liu ${ }^{1,2}$, Haining Cui ${ }^{1 *}$ and Ying $X u^{2,3 *}$ \\ ${ }^{1}$ College of Physics, Jilin University, Changchun, China, ${ }^{2}$ Department of Biochemistry and Molecular Biology, Institute of \\ Bioinformatics, The University of Georgia, Athens, GA, United States, ${ }^{3}$ Cancer Systems Biology Center, The China-Japan \\ Union Hospital, Jilin University, Changchun, China
}

Quantitative assessment of the intracellular oxidative stress level is a very important problem since it is the basis for elucidation of the fundamental causes of metabolic changes in diseased human cells, particularly cancer. However, the problem proves to be very challenging to solve in vivo because of the complex nature of the problem. Here a computational method is presented for predicting the quantitative level of the intracellular oxidative stress in cancer tissue cells. The basic premise of the predictor is that the genomic mutation level is strongly associated with the intracellular oxidative stress level. Based on this, a statistical analysis is conducted to identify a set of enzyme-encoding genes, whose combined expression levels can well explain the mutation rates in individual cancer tissues in the TCGA database. We have assessed the validity of the predictor by assessing it against genes that are known to have anti-oxidative functions for specific types of oxidative stressors. Then the applications of the predictor are conducted to illustrate its utility.

Keywords: oxidative stress, genomic mutation, transcriptomic data, cancer, TCGA data analysis, computational prediction

\section{INTRODUCTION}

Oxidative stress has long been recognized as a cellular stress associated with cancer formation and development (Cerutti and Trump, 1993; Wangpaichitr et al., 2009; Bellezza et al., 2010; Chan et al., 2010; Conti et al., 2010; Dayem et al., 2010; Gibellini et al., 2010; Henkler et al., 2010; Karlenius and Tonissen, 2010; Massi et al., 2010; Ortega et al., 2010; Pizzimenti et al., 2010; Reuter et al., 2010; Schultz et al., 2010; Soory, 2010; Sun et al., 2010; Valle et al., 2010; Gorrini et al., 2013; Sosa et al., 2013; Leone et al., 2017). It refers to the gap between the intracellular oxidizing power and the anti-oxidation capacity in a human cell. Numerous authors have pointed out that oxidative stress may be responsible for the induction of a variety of altered metabolisms in cancer, which include (i) considerably increased lipid metabolism (Santos and Schulze, 2012; Zhang and Du, 2012; Alfaradhi et al., 2014; Zhang et al., 2014), (ii) altered metabolisms of sulfur-containing amino acids (Schulz et al., 2000; Zhang et al., 2012; Campbell et al., 2016; Martínez et al., 2017), (iii) reprogrammed sugar metabolisms (Takeyama et al., 2000; Wu et al., 2009; Dewald et al., 2016), among other reprogrammed metabolisms. Increased genomic mutations in cancer have also been 
attributed to oxidative stress (Limoli and Giedzinski, 2003; Doudican et al., 2005; Slane et al., 2006; Xu et al., 2014; Fitzgerald et al., 2017; Markkanen, 2017; Rao et al., 2017; Tubbs and Nussenzweig, 2017). However, it has proven to be very challenging to accurately estimate the level of intracellular oxidative stress (Selvaraj et al., 2002; Farah, 2005). Because existing techniques are mostly designed to detect quantitatively specific oxidizing molecular species (e.g., $\mathrm{H}_{2} \mathrm{O}_{2}$ and $\mathrm{O}_{2}$ ) rather than detecting the overall level of oxidative stress. Computational techniques could potentially play a key role in estimating the quantitative level of oxidative stress.

Unlike hypoxia or acid-base imbalance whose related stress can be measured/estimated in terms of the concentration of one or a few molecular species such as $\mathrm{O}_{2}$ or $\mathrm{H}+$, there are many types of oxidizing molecules such as reactive oxygen species (Barash et al., 2010; Reczek and Chandel, 2017), reactive nitrogen species (Fionda et al., 2016; Kruk and Aboul-Enein, 2017), reactive lipid species (Higdon et al., 2012; Graham, 2016) among others. Furthermore, to estimate the stress level, it also requires to know the total cellular reducing capacity. This is also challenging since human cells not only have a basic set of anti-oxidation (or reducing) capacities consisting of (1) glutathione and associated enzymes (e.g., glutathione transferase and peroxidase), (2) vitamin A, C, E and derivatives like beta-carotene, and (3) anti-oxidation enzymes like SOD (superoxide dismutase), PRX (periaxin), and TRX (thioredoxin) (Chang et al., 2008; Mantovani et al., 2010), but also rely on antioxidation capacities of various other molecules including some enzymes and fatty acids. Among them, the main functions of the enzymes may not be for anti-oxidation (Osmundsen et al., 1982; Wieczorek et al., 2017), while the anti-oxidative properties of fatty acids have long been well-established (Richard et al., 2008; Freitas et al., 2017). All these make it very difficult to pin down on what molecular species should be used when assessing the level of oxidative stress.

A few proposals have been made regarding possible biomarkers for intracellular level of oxidative stress such as the carbonylation level (aldehydes and ketones) of proteins (DalleDonne et al., 2003; Hacişevki et al., 2012; Fernando et al., 2016), the level of oxidized low-density lipoprotein (Itabe, 2012; Osman et al., 2016), oxidized products of lipids such as 4HNE (4-hydroxynonenal) and MDA (malondialdehyde) (Niki, 2008; Halder and Bhattacharyya, 2014; Teppner et al., 2015), and protein thiols (Giustarini et al., 2012, 2017). There are two general issues with these biomarkers: (1) they tend to reflect the level of oxidation by specific oxidizing molecules; and (2) more importantly, they are not high-throughput, hence it is impractical for large-scale analyses, such as analyses of TCGA tissue samples (https://portal.gdc.cancer.gov/) to elucidate possible causes of specific metabolic changes in such tissues.

The goal here is to identify a set of genes whose mRNA expression levels can collectively reflect the overall level of intracellular oxidative stress present in a cancer tissue. The strategy is: (1) the somatic point-mutation rate in each cancer tissue sample is used as an indicator for the level of intracellular oxidative stress, an idea that has been well-established and applied (Doudican et al., 2005; Slane et al., 2006; Xu et al., 2014;
Fitzgerald et al., 2017; Markkanen, 2017; Rao et al., 2017; Tubbs and Nussenzweig, 2017); (2) a few enzyme classes are selected, which are known to have anti-oxidation activities such as EC 3.1.-, EC 3.6.-, EC 2.4.-, and EC 2.7.- (Kato et al., 1995; Kong et al., 2007); and (3) a subset of genes are selected from these enzyme classes, whose combined expressions correlate strongly with the mutation rates in the matching genomes, determined through regression analyses. One assumption used in the analysis is that the oxidative stress levels in cytosol and nucleus are the same, which is reasonable knowing that the nuclear pores are large enough to allow most of the oxidizing molecules to go through freely between the two compartments; and increased mutation rates in cancer are known to be related to the nucleus oxidative stress level (Chung et al., 2014; Markkanen, 2017).

By applying this strategy to gene-expression data and matching genomic mutation data of cancer tissues of 14 cancer types in TCGA, representing all those with sufficiently large sample sizes, we have trained a predictor for the intracellular level of oxidative stress for each of the 14 cancer types. They are BLCA (bladder urothelial carcinoma), BRCA (breast invasive carcinoma), COAD (colon adenocarcinoma), ESCA (esophageal carcinoma), HNSC (head and neck squamous cell carcinoma), $\mathrm{KICH}$ (kidney renal papillary cell carcinoma), KIRC (kidney chromophobe), KIRP (kidney renal clear cell carcinoma), LICH (liver hepatocellular carcinoma), LUAD (lung squamous cell carcinoma), LUSC (lung adenocarcinoma), PRAD (prostate adenocarcinoma), STAD (stomach adenocarcinoma), and THCA (thyroid carcinoma). We have then validated the predictor against data with known oxidative stress related information. A key advantage in having a gene-expression data-based predictor is that RNA-seq data tend to be collected in general for cancer research; and such a predictor does not require a user to know the mutation rate distribution of the cancer type under study since such information is already encoded in the predictor for each cancer type.

\section{RESULTS}

\section{Genomic Mutation Profiles}

We have calculated and plotted the distribution of the number of point-mutations in coding regions per genome across all cancer genomes for each of the 14 cancer types, as shown in Figure 1. From the figure, LUAD has the highest average mutation rate at 347.20 per genome, and $\mathrm{KICH}$ has the lowest one at 19.75 per genome. The following lists the average numbers of the remaining 12 cancer types in the descending order: 288.95 mutations in LUSC, 272.02 in COAD, 257.50 in STAD, 234.71 in BLCA, 213.26 in HNSC, 124.59 in ESCA, 147.65 in LIHC, 303.75 in BRCA, 111.14 in KIPR, 113.94 in KIRC, 138.75 in PRAD, and 134.18 in THCA.

\section{Selection of Antioxidant Enzyme-Encoding Genes}

Some classes of enzymes have long been known to have anti-oxidative functions as their main function such as the antioxidant enzymes mentioned earlier while some others have anti-oxidation as their secondary function such as cytochromes 


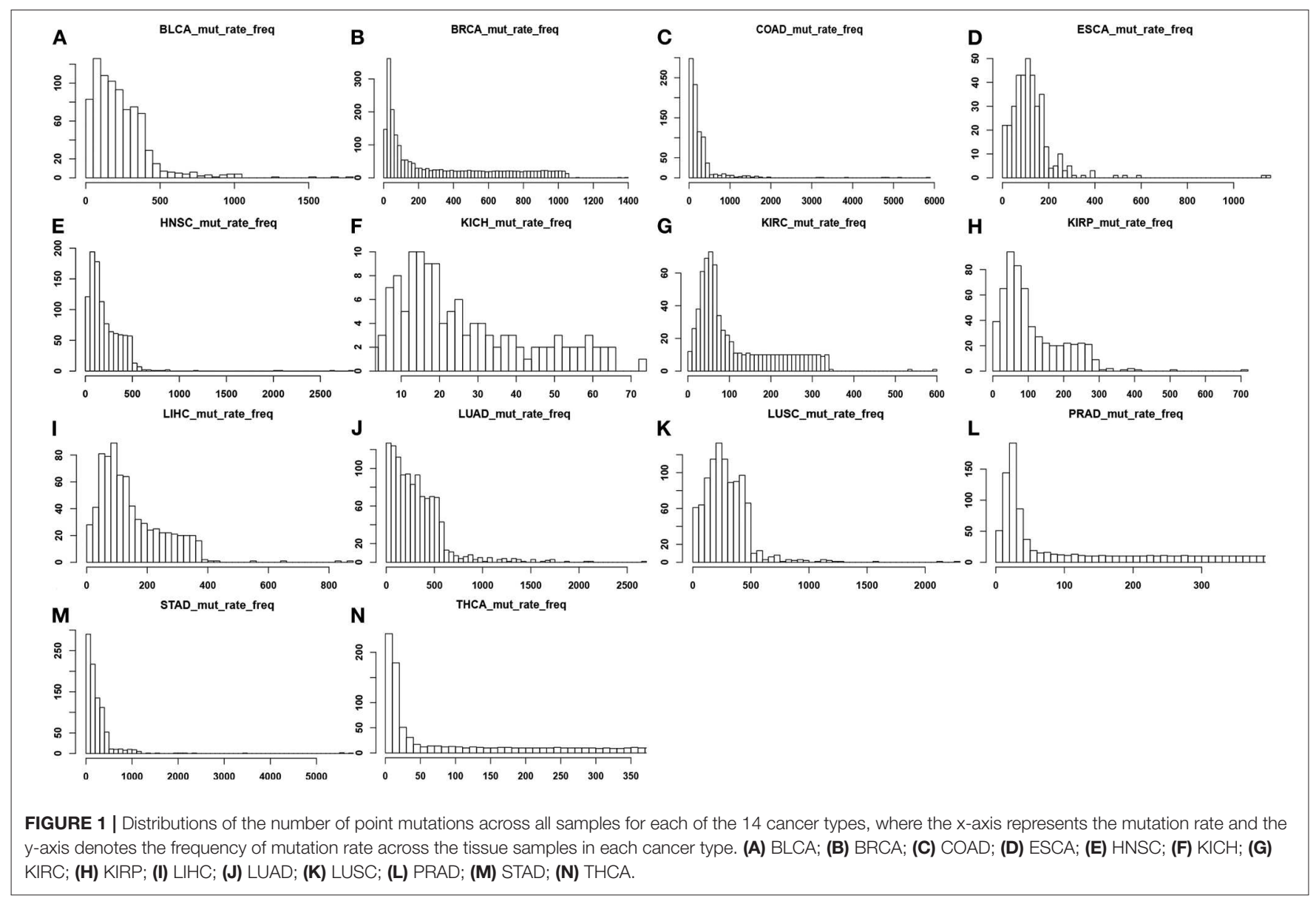

P450 and mitogen activated protein kinases (Limón-Pacheco and Gonsebatt, 2009). And yet, increasingly more proteins have been found to have antioxidant roles in addition to their main functions such as translocases (Tang et al., 2017), hydrolase (Liu et al., 2018), and hexokinase (Heneberg, 2019).

Based on such information, we have conducted a preliminary regression analysis of the mutation rates against the expression data of all the enzyme-encoding genes in the same samples for each of the 14 cancer types (with a Lasso penalty). Interestingly, genes that give rise to good regression results across all 14 cancer types generally fall into a small set of enzyme subclasses, particularly EC 3.- (hydrolases) and EC 2.- (transferases). Hence, we have conducted a second round of regression against expression data of genes only in these two EC classes.

From genes selected for each of the 14 regression models, we note the following: (1) in all 14 cancer types, genes in each regression model fall into exactly four sub-subclasses of EC 2.and EC 3.-; (2) in five cancer types: BLCA, KIRC, KIRP, LUAD, and LUSC, all the genes fall into four sub-subclasses of EC 3.-; in three cancer types, BRCA, ESCA and $\mathrm{KICH}$, all genes fall into four sub-subclasses of EC 2.-; and in the remaining six cancer types: COAD, HNSC, LIHC, PRAD, STAD, and THCA, all genes fall into two subclasses of EC 2.- and two subclasses of EC 3.-; (3) the two most commonly used EC 3.- subclasses are EC 3.4.21.and EC 3.1.3.- while the two mostly used EC 2.- subclasses are
EC 2.4.1- and EC 2.7.1.-. Table $\mathrm{S} 1$ gives the gene names selected in the regression model for each cancer type.

\section{Linear Regression Analyses}

For each cancer type, a linear regression model is trained to predict the mutation rate using expression data in the same cancer sample, of selected genes from some EC subclasses as discussed above. The detailed objective function is described in the Methods section. To ensure the quality of each trained model, we have randomly selected $2 / 3$ of the samples as the training data, and used the remaining $1 / 3$ as the test data. Figure 2 shows the predicted values by the best trained model vs. the actual mutation rates across all samples for each cancer type. Similar plots for the test samples are given in Figure S1. Table 1 summarizes the level of contribution by genes of each EC subclass to the regression result for each cancer type, with the detailed information of the 14 models being given in Table S2.

\section{Prediction Validation}

We have conducted the following analyses to provide supporting data to our prediction model.

\section{Validation Against Fatty Acid Synthesis Genes}

It is well-known that fatty acids serve as antioxidants in cancer and other disease cells (Richard et al., 2008; Freitas et al., 2017). 


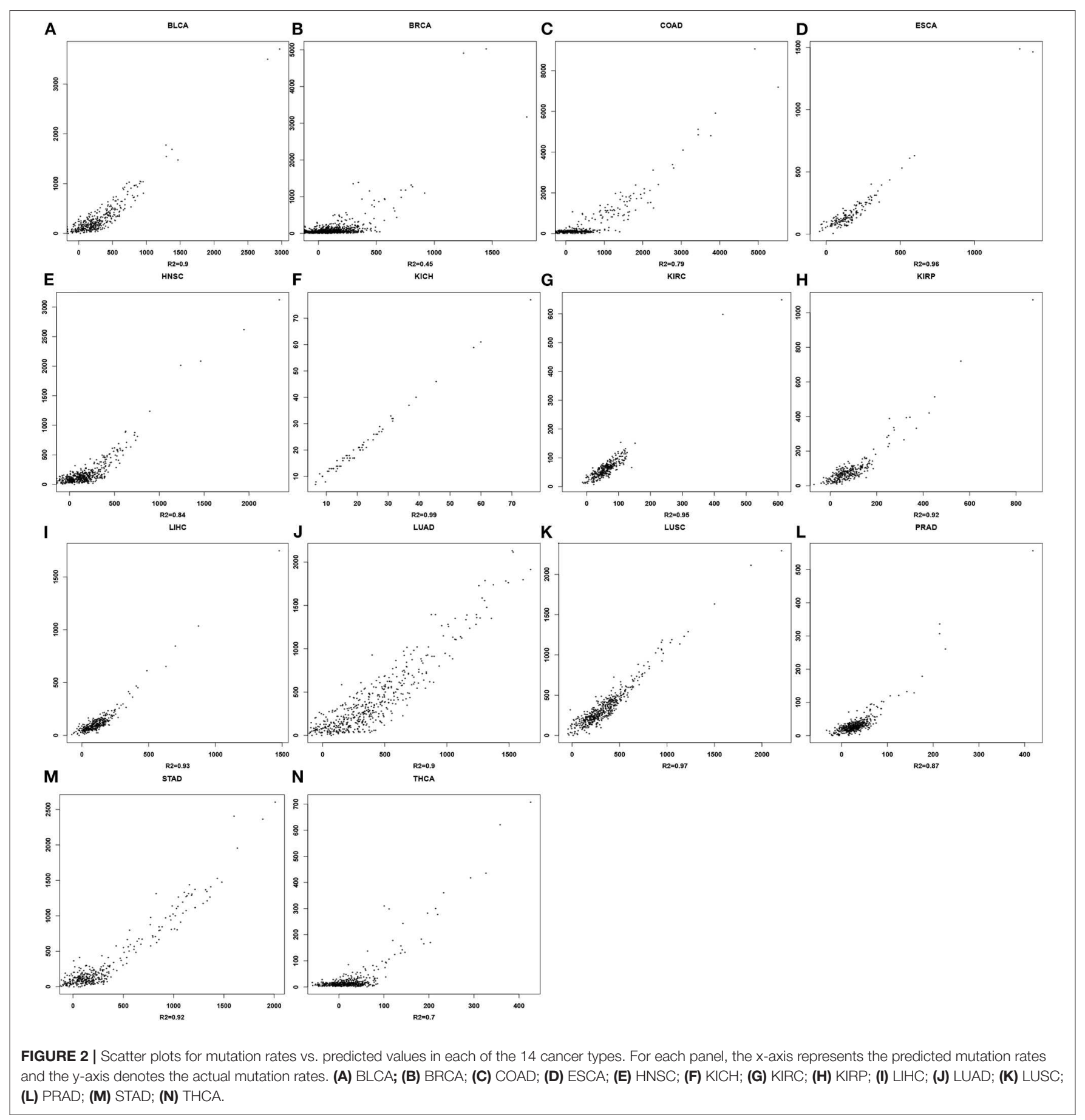

Hence, we anticipate that our predictor should have some level of correlation with the fatty acid synthesis process. Table 2 shows the best correlation coefficient between our predictor and one of the four fatty acid synthesis genes (see Methods) across the 14 cancer types, with Table 3 giving the statistical significance of the observed correlations.

\section{Validation Against Mucin Genes}

Mucins have been found to have elevated levels across numerous cancer types and are known to have anti-oxidation roles (Takeyama et al., 2000; Wu et al., 2009; Dewald et al., 2016). We have examined their expression levels and our predictor. Table 4 shows the best correlation coefficient between our predictor and one of the mucin genes across the 14 cancer types, with Table 5 giving the statistical significance of the observed correlations. And the statistical significance of the observed correlations for each Mucin genes is given in Table S3 in detail.

\section{Validation Against Glutathione Synthesis Genes}

Glutathione is a molecule human cells use as a key antioxidant. We have examined their expression levels and our predictor. Table 6 shows the R2 values between our predictor and 
TABLE 1 | Estimated contribution by genes in each EC subclass to the linear model for each of the 14 cancer types.

\begin{tabular}{|c|c|c|}
\hline Cancer name & EC subclasses used & Contributions by each EC class \\
\hline \multirow[t]{4}{*}{ BLCA } & 3.1 .1 & 2.06E-22 \\
\hline & 3.1 .4 & $2.02 E-26$ \\
\hline & 3.4 .21 & $4.88 \mathrm{E}-13$ \\
\hline & 3.2 .1 & $1.46 \mathrm{E}-21$ \\
\hline \multirow[t]{4}{*}{ BRCA } & 2.3 .1 & $3.52 E-21$ \\
\hline & 2.4 .1 & $2.11 \mathrm{E}-24$ \\
\hline & 2.7 .1 & $2.41 \mathrm{E}-14$ \\
\hline & 2.7 .7 & $2.65 E-18$ \\
\hline \multirow[t]{4}{*}{ COAD } & 2.7 .1 & 1.18E-14 \\
\hline & 2.4 .1 & $5.97 \mathrm{E}-13$ \\
\hline & 3.1 .3 & $2.44 \mathrm{E}-20$ \\
\hline & 3.4 .21 & $3.02 \mathrm{E}-16$ \\
\hline \multirow[t]{4}{*}{ ESCA } & 2.1 .1 & 4.55E-71 \\
\hline & 2.3 .1 & 7.00E-04 \\
\hline & 2.4 .1 & 5.07E-07 \\
\hline & 2.6 .1 & $9.62 \mathrm{E}-22$ \\
\hline \multirow[t]{4}{*}{ HNSC } & 2.7 .1 & $2.94 \mathrm{E}-13$ \\
\hline & 2.4 .1 & 7.11E-19 \\
\hline & 3.1 .3 & $5.58 \mathrm{E}-14$ \\
\hline & 3.4 .21 & $2.51 \mathrm{E}-16$ \\
\hline \multirow[t]{4}{*}{$\mathrm{KICH}$} & 2.7 .7 & 2.09E-05 \\
\hline & 2.1 .1 & $1.78 \mathrm{E}-16$ \\
\hline & 2.5 .1 & 4.37E-05 \\
\hline & 2.8 .2 & 6.96E-06 \\
\hline \multirow[t]{4}{*}{ KIRC } & 3.1 .1 & $1.16 \mathrm{E}-10$ \\
\hline & 3.1 .3 & $9.65 \mathrm{E}-17$ \\
\hline & 3.2 .1 & $2.20 \mathrm{E}-10$ \\
\hline & 3.4 .21 & 5.91E-28 \\
\hline \multirow[t]{4}{*}{$\mathrm{KIRP}$} & 3.1 .1 & $8.78 \mathrm{E}-14$ \\
\hline & 3.1 .3 & 6.68E-07 \\
\hline & 3.4 .21 & 1.60E-23 \\
\hline & 3.6 .3 & $6.95 \mathrm{E}-16$ \\
\hline \multirow[t]{4}{*}{ LIHC } & 2.4 .1 & $1.41 \mathrm{E}-21$ \\
\hline & 3.1 .3 & $1.44 \mathrm{E}-12$ \\
\hline & 3.4 .21 & $2.33 E-12$ \\
\hline & 2.3 .1 & 6.07E-25 \\
\hline \multirow[t]{4}{*}{ LUAD } & 3.1 .3 & 1.65E-63 \\
\hline & 3.1 .4 & 3.48E-07 \\
\hline & 3.4 .21 & $5.55 E-18$ \\
\hline & 3.6 .3 & 5.10E-35 \\
\hline \multirow[t]{4}{*}{ LUSC } & 3.1 .1 & $5.77 E-43$ \\
\hline & 3.1 .3 & 2.09E-24 \\
\hline & 3.4 .21 & $1.78 \mathrm{E}-26$ \\
\hline & 3.6 .3 & 8.40E-22 \\
\hline \multirow[t]{4}{*}{ PRAD } & 2.7 .1 & $2.74 \mathrm{E}-41$ \\
\hline & 2.4 .1 & $1.66 \mathrm{E}-11$ \\
\hline & 3.4 .21 & $5.10 \mathrm{E}-14$ \\
\hline & 3.1 .3 & 5.27E-05 \\
\hline \multirow[t]{4}{*}{ STAD } & 2.7 .1 & 5.47E-23 \\
\hline & 2.4 .1 & $1.34 \mathrm{E}-15$ \\
\hline & 3.1 .3 & 5.06E-16 \\
\hline & 3.4 .21 & 8.40E-08 \\
\hline \multirow[t]{4}{*}{ THCA } & 2.7 .1 & 2.27E-31 \\
\hline & 2.4 .1 & $5.53 \mathrm{E}-12$ \\
\hline & 3.1 .3 & $6.47 \mathrm{E}-11$ \\
\hline & 3.4 .21 & 1.05E-06 \\
\hline
\end{tabular}

TABLE 2 | Correlation coefficients between our oxidative-stress predictor and the fatty acid synthesis gene across 14 cancer types.

\begin{tabular}{lll}
\hline Cancer name & Correlation coefficient & Gene name \\
\hline BLCA & 0.77 & FASN \\
BRCA & 0.64 & ACAT2 \\
COAD & 0.89 & ACAT1 \\
ESCA & 0.99 & ACAT2 \\
HNSC & 0.92 & MCAT \\
KICH & 0.98 & ACAT2 \\
KIRC & 0.68 & ACAT1 \\
KIRP & 0.8 & ACAT1 \\
LIHC & 0.73 & FASN \\
LUAD & 0.81 & ACAT2 \\
LUSC & 0.69 & FASN \\
PRAD & 0.72 & FASN \\
STAD & 0.66 & MCAT \\
THCA & 0.79 & ACAT1 \\
\hline
\end{tabular}

TABLE 3 | Statistical significance for the observed correlation coefficient in Table 2 across 14 cancer types.

\begin{tabular}{lllll}
\hline Cancer name & ACAT1 & MCAT & ACAT2 & FASN \\
\hline BLCA & $1.43 \mathrm{E}-01$ & $1.44 \mathrm{E}-01$ & $8.39 \mathrm{E}-02$ & $4.36 \mathrm{E}-02$ \\
BRCA & $2.60 \mathrm{E}-04$ & $9.02 \mathrm{E}-02$ & $7.97 \mathrm{E}-15$ & $1.64 \mathrm{E}-02$ \\
COAD & $1.10 \mathrm{E}-03$ & $2.88 \mathrm{E}-06$ & $8.34 \mathrm{E}-05$ & $3.07 \mathrm{E}-01$ \\
ESCA & $8.21 \mathrm{E}-01$ & $8.18 \mathrm{E}-01$ & $4.84 \mathrm{E}-01$ & $8.06 \mathrm{E}-01$ \\
HNSC & $4.30 \mathrm{E}-01$ & $1.89 \mathrm{E}-09$ & $2.43 \mathrm{E}-01$ & $5.64 \mathrm{E}-02$ \\
KICH & $1.29 \mathrm{E}-01$ & $5.44 \mathrm{E}-01$ & $8.24 \mathrm{E}-03$ & $9.24 \mathrm{E}-01$ \\
KIRC & $4.27 \mathrm{E}-03$ & $3.35 \mathrm{E}-02$ & $2.04 \mathrm{E}-01$ & $1.42 \mathrm{E}-01$ \\
KIRP & $1.55 \mathrm{E}-03$ & $6.18 \mathrm{E}-02$ & $7.80 \mathrm{E}-01$ & $9.07 \mathrm{E}-02$ \\
LIHC & $5.70 \mathrm{E}-02$ & $5.22 \mathrm{E}-01$ & $4.62 \mathrm{E}-01$ & $2.81 \mathrm{E}-04$ \\
LUAD & $9.87 \mathrm{E}-01$ & $5.16 \mathrm{E}-03$ & $5.63 \mathrm{E}-05$ & $2.12 \mathrm{E}-05$ \\
LUSC & $8.29 \mathrm{E}-01$ & $4.06 \mathrm{E}-01$ & $4.05 \mathrm{E}-02$ & $3.48 \mathrm{E}-02$ \\
PRAD & $3.38 \mathrm{E}-02$ & $9.83 \mathrm{E}-01$ & $9.33 \mathrm{E}-07$ & $2.02 \mathrm{E}-01$ \\
STAD & $8.52 \mathrm{E}-01$ & $6.19 \mathrm{E}-04$ & $8.79 \mathrm{E}-03$ & $7.84 \mathrm{E}-01$ \\
THCA & $2.87 \mathrm{E}-02$ & $2.97 \mathrm{E}-01$ & $1.60 \mathrm{E}-01$ & $1.86 \mathrm{E}-01$ \\
\hline
\end{tabular}

the glutathione synthesis genes across the 14 cancer types, with Table 7 giving the statistical significance of the observed correlations.

It is noteworthy that our predictor is designed to predict the level of oxidative stress; and each of the above three groups of genes is known to be associated with the level of oxidative stress and not involved in the training dataset. The observed strong correlations between our predicted oxidative stress levels and the expression levels of each such group, along with significant $p$ values, provide strong support for that our predictor captures the anti-oxidative level from different independent aspects, hence indicating the validity of our trained predictor as an indicator for the level of oxidative stress.

\section{Application}

To demonstrate the utility of our predictor, we have calculated the average oxidative stress levels for each of the four stages 
TABLE 4 | Correlation coefficients between our oxidative-stress predictor and mucin genes across 14 cancer types.

\begin{tabular}{lcl}
\hline Cancer name & Correlation coefficient & Gene name \\
\hline BLCA & 0.69 & MUC15 \\
BRCA & 0.8 & MUC20 \\
COAD & 0.75 & MUC5B \\
ESCA & 0.87 & MUC17 \\
HNSC & 0.86 & MUC3A \\
KICH & 0.91 & MUC12 \\
KIRC & 0.83 & MUC16 \\
KIRP & 0.97 & MUC12 \\
LIHC & 0.72 & MUC20 \\
LUAD & 0.94 & MUC22 \\
LUSC & 0.92 & MUC4, MUC20 \\
PRAD & 0.76 & MUC6 \\
STAD & 0.88 & MUC7 \\
THCA & 0.88 & MUC15 \\
\hline
\end{tabular}

TABLE 5 | Statistical significance for the observed correlation coefficients in Table 4 across 14 cancer types.

\begin{tabular}{lll}
\hline Cancer name & Statistics significance & Gene name \\
\hline BLCA & $2.40 \mathrm{E}-13$ & MUC15 \\
BRCA & $4.01 \mathrm{E}-11$ & MUC20 \\
COAD & $2.04 \mathrm{E}-12$ & MUC5B \\
ESCA & $8.74 \mathrm{E}-07$ & MUC17 \\
HNSC & $8.74 \mathrm{E}-07$ & MUC3A \\
KICH & $1.10 \mathrm{E}-04$ & MUC12 \\
KIRC & $3.95 \mathrm{E}-07$ & MUC6 \\
KIRP & $9.38 \mathrm{E}-06$ & MUC12 \\
LIHC & $2.96 \mathrm{E}-08$ & MUC1 \\
LUAD & $6.28 \mathrm{E}-07$ & MUC22 \\
LUSC & $8.48 \mathrm{E}-11$ & MUC1 \\
PRAD & $2.85 \mathrm{E}-04$ & MUC6 \\
STAD & $3.40 \mathrm{E}-07$ & MUC17 \\
THCA & $2.27 \mathrm{E}-03$ & MUC15 \\
\hline
\end{tabular}

vs. the matching controls in each of the 14 cancer types, as detailed in Figure 3. From the figure, we can see: (1) cancer tissue cells have elevated oxidative-stress levels than matching controls in all of 14 cancer types; and (2) the oxidative stress level tends to progressively increase as a cancer advances from stage 1 through stage 4 , in 10 out of 14 cancer types with at most one predicted level of oxidative stress being out of order. This is clearly consistent with our general understanding about cancer progression.

We have also conducted co-expression analyses to find genes that strongly correlate with our predictor in each cancer type and analyzed the pathways enriched by such genes. Table $\mathbf{S} 4$ gives the up-to top 100 enriched pathways with $p<0.05$ in each cancer. We note that the enriched pathways are highly consistent across different cancer types. Furthermore, we note based on extensive literature search that a majority of the enriched pathways, marked in bold letters, is: (1) involved in anti-oxidation activities, (2)
TABLE 6 | Correlation coefficients between our predictor and the glutathione synthesis genes across 14 cancer types.

\begin{tabular}{lcl}
\hline Cancer name & Correlation coefficient & Gene name \\
\hline BLCA & 0.63 & GCLM \\
BRCA & 0.57 & GCLM \\
COAD & 0.94 & GSS \\
ESCA & 0.78 & GCLC \\
HNSC & 0.77 & GCLM \\
KICH & 0.45 & GCLC \\
KIRC & 0.74 & GSS \\
KIRP & 0.75 & GSS \\
LIHC & 0.66 & GCLC \\
LUAD & 0.74 & GCLM \\
LUSC & 0.75 & GSS \\
PRAD & 0.82 & GCLC \\
STAD & 0.79 & GCLM \\
THCA & 0.75 & GCLC \\
\hline
\end{tabular}

TABLE 7 | Statistical significance for observed correlation coefficient in Table 6 across 14 cancer types.

\begin{tabular}{lccc}
\hline Cancer name & GCLC & GSS & GCLM \\
\hline BLCA & $3.56 \mathrm{E}-01$ & $7.67 \mathrm{E}-03$ & $1.12 \mathrm{E}-07$ \\
BRCA & $3.07 \mathrm{E}-01$ & $4.49 \mathrm{E}-01$ & $5.16 \mathrm{E}-09$ \\
COAD & $8.20 \mathrm{E}-01$ & $1.53 \mathrm{E}-05$ & $3.14 \mathrm{E}-02$ \\
ESCA & $3.61 \mathrm{E}-14$ & $4.09 \mathrm{E}-01$ & $5.25 \mathrm{E}-01$ \\
HNSC & $4.62 \mathrm{E}-01$ & $3.05 \mathrm{E}-04$ & $1.47 \mathrm{E}-06$ \\
KICH & $1.38 \mathrm{E}-01$ & $9.81 \mathrm{E}-01$ & $7.89 \mathrm{E}-01$ \\
KIRC & $5.90 \mathrm{E}-01$ & $3.65 \mathrm{E}-05$ & $3.67 \mathrm{E}-03$ \\
KIRP & $7.88 \mathrm{E}-01$ & $3.42 \mathrm{E}-07$ & $7.88 \mathrm{E}-01$ \\
LIHC & $5.50 \mathrm{E}-01$ & $6.13 \mathrm{E}-06$ & $9.30 \mathrm{E}-02$ \\
LUAD & $1.88 \mathrm{E}-03$ & $3.17 \mathrm{E}-02$ & $6.50 \mathrm{E}-02$ \\
LUSC & $4.62 \mathrm{E}-02$ & $2.50 \mathrm{E}-29$ & $3.15 \mathrm{E}-01$ \\
PRAD & $1.11 \mathrm{E}-01$ & $7.47 \mathrm{E}-02$ & $4.29 \mathrm{E}-04$ \\
STAD & $3.24 \mathrm{E}-04$ & $1.74 \mathrm{E}-07$ & $1.21 \mathrm{E}-08$ \\
THCA & $9.80 \mathrm{E}-04$ & $1.92 \mathrm{E}-01$ & $2.08 \mathrm{E}-02$ \\
\hline
\end{tabular}

induced by oxidative stress, (3) induces oxidative stress, (4) involved in reactive oxidative stress signaling, and/or (5) is altered by oxidative stress. While these data provide supporting data to our predictor, pathways not known to be oxidative stress related may provide useful candidates for elucidation of the overall oxidative-stress responding mechanisms in cancer cells.

\section{DISCUSSION}

Quantitative assessment of the intracellular oxidative stress will prove to be an invaluable tool for elucidation of the possible causes of various changes in cancer cells, including extensive metabolic reprogramming. However, the problem proves to be very challenging because there are large numbers of contributors to both the total oxidizing power and the anti-oxidizing capacity in human cells. Previous studies tend to focus on oxidative stress 


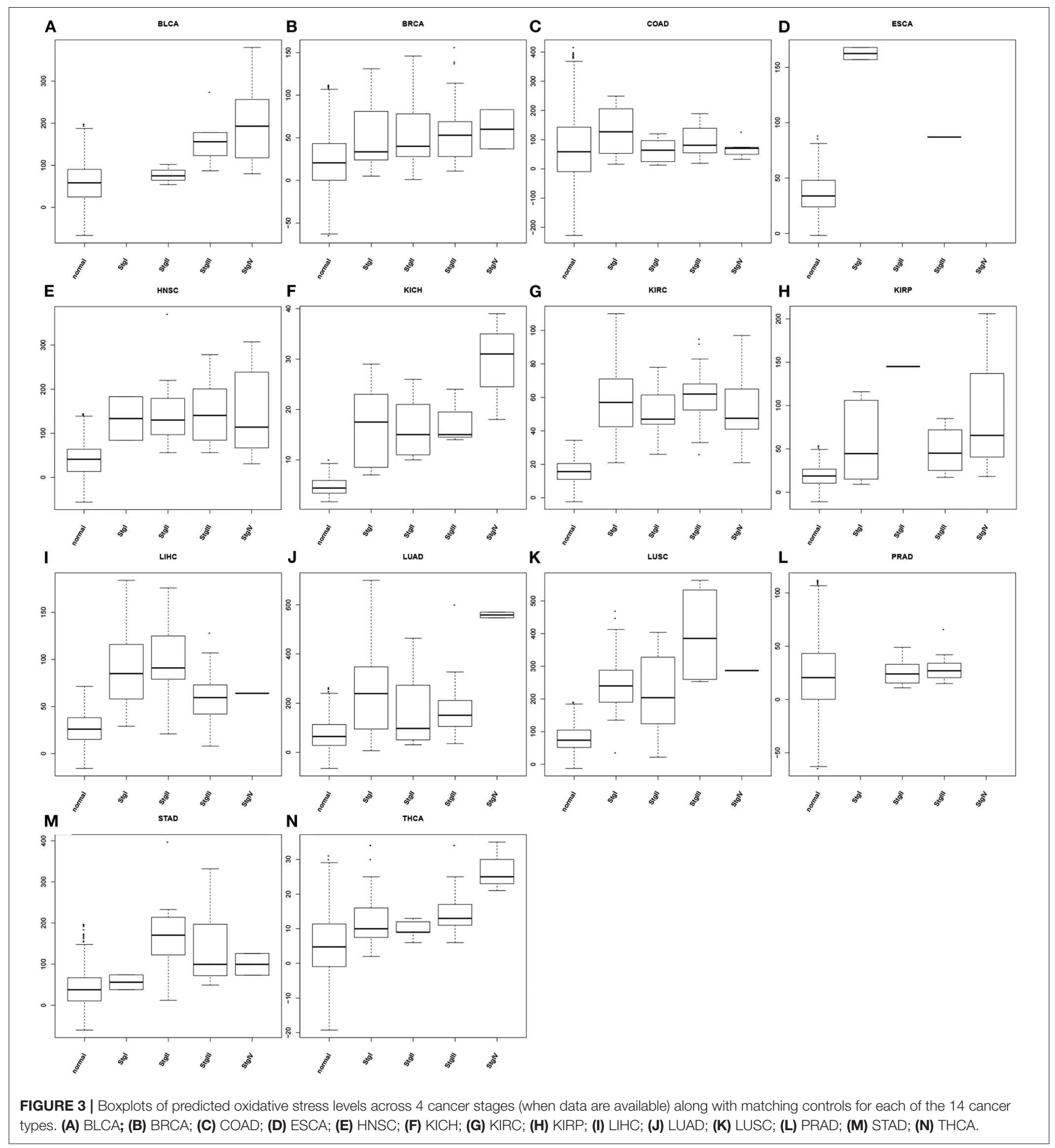

induced by specific molecular species such as $\mathrm{H}_{2} \mathrm{O}_{2}$ or lipid radicals rather than the overall oxidative stress level. To the best our knowledge, our work is the first computational tool for estimating the oxidative stress level. The basic premise of our tool is that the genomic mutation level is strongly associated with the overall intracellular oxidative stress level. The second premise is that many enzymes have anti-oxidation capacity as reported by numerous authors (Sies, 1997; Rajput et al., 2013).

One surprising result in our regression analyses is that genes in two EC classes, EC 2.- (transferases) and EC 3.(hydrolases), specifically four subclasses of these two, can be used to well interpret the mutation rate in each cancer sample by 
a linear combination of their expressions with high statistical significance. This strongly suggests that all the enzymes encoded by these genes play roles in cellular anti-oxidation, which is clearly a novel discovery and warrants further investigation. Furthermore, different cancer types tend to use a distinct combination of genes from four subclasses of enzymes strongly suggest that these cancer types may encounter different types of oxidative stress, hence using different combinations of antioxidation enzymes. This also warrants further study regarding why genes in different enzyme classes show strong correlations with mutation rates in different cancer types, therefore to understand the detailed mechanisms of their anti-oxidative functions possibly for different types of oxidants.

As mentioned in the Introduction, oxidative stress may arise from different molecular species such reactive oxygen species, reactive nitrogen species, reactive lipid species and various free radicals. Different molecular species might be utilized to consume specific types of oxidizing molecules. By showing that three independent classes of molecules all have statistically significant correlation coefficients with our general-purpose predictor for oxidative stress, we clearly have strong support for the validity of our predictor.

The availability of this new tool make open new doors for studying impact of oxidative stress on various chronic inflamed diseases, including cancer, including (i) elucidation of all metabolic processes, particularly reprogrammed metabolisms observed in cancer and other diseases, that are statistically associated with the level of oxidative stress, hence providing a new capability for detection of the possible causes of various altered metabolisms, and (ii) systematic analyses of different classes of enzymes in terms of their anti-oxidative roles, which could provide potentially powerful and new targets for treating various chronic diseases, including cancer.

We anticipate that our predictor will prove a powerful tool useful for elucidation of causes of variety of systematic changes, including metabolic reprogramming to gain indepth understanding of why specific metabolic pathways are reprogrammed and certain cellular functions tend to be repressed or hyper-activated in cancer.

\section{DATA AND METHODS}

\section{Gene-Expression Data and Genomic Mutation Data}

Gene expression and genomic mutation data of 14 cancer types: BLCA, BRCA, COAD, ESCA, HNSC, KICH, KIRC, KIRP, LIHC, LUAD, LUSC, PRAD, STAD, and THCA were downloaded from the TCGA data portal ${ }^{1}$. The detailed information of these data is summarized in Table 8.

Genomic mutation data were derived from the wholeexome sequencing data. Specifically, somatic changes are identified through comparing allele frequencies in the aligned DNA sequences of each cancer and the matching normal samples, using the GDC DNA-Seq analysis pipeline (GDC DNA-Seq analysis pipeline: https://docs.gdc.cancer.gov/

\footnotetext{
${ }_{1}^{1}$ TCGA data portal: https://portal.gdc.cancer.gov/
}

TABLE 8 | Information for 14 cancer types.

\begin{tabular}{lcc}
\hline Cancer name & Number of cancer samples & Number of control samples \\
\hline BLCA & 408 & 20 \\
BRCA & 1,092 & 114 \\
COAD & 456 & 42 \\
ESCA & 164 & 12 \\
HNSC & 501 & 45 \\
KICH & 66 & 25 \\
KIRC & 530 & 73 \\
KIRP & 289 & 33 \\
LICH & 371 & 51 \\
LUAD & 515 & 60 \\
LUSC & 501 & 50 \\
PRAD & 495 & 53 \\
STAD & 380 & 33 \\
THCA & 502 & 59 \\
& &
\end{tabular}

Data/Bioinformatics_Pipelines/DNA_Seq_Variant_Calling_ Pipeline). MuTect2 (GDC MuTect2: https://docs.gdc.cancer. gov/Data/Bioinformatics_Pipelines/DNA_Seq_Variant_Calling_ Pipeline/\#somatic-variant-calling-workflow) is used to call each mutation using the default parameters.

Human enzyme information, including gene names and the relevant enzyme classes, is downloaded from BRENDA (BRENDA links: https://www.brenda-enzymes.org/).

\section{Synthesis of Fatty Acids}

We have used the four fatty-acid synthesis genes: FASN (Fatty Acid Synthase), ACAT1 (Acetyl-CoA Acetyltransferase 1), ACAT2 (Acetyl-CoA Acetyltransferase 2), and MCAT (MalonylCoA-Acyl Carrier Protein Transacylase) to reflect the level of fatty acid synthesis, and have calculated the Pearson correlation coefficient between the expressions of one of them and our predictor.

\section{Mucin Genes}

Human has 20 mucin genes, namely MUC1, MUC2, MUC8, MUC12, MUC13, MUC15, MUC16, MUC17, MUC19, MUC20, MUC21, MUC22, MUC3A, MUC3B, MUC4, MUC5AC, MUC5B, MUC6, MUC7, and MUCL1. To assess their overall correlation with our predictor, we have conducted the Pearson correlation coefficient between the expression of each mucin gene and our oxidative stress predictor.

\section{Glutathione Synthesis}

We have used the three biosynthesis genes: GCLC (glutamatecysteine ligase catalytic subunit), GCLM (glutamate-cysteine ligase modifier subunit) and GSS (glutathione synthase) to reflect the level of glutathione synthesis. As above, we have calculated the Pearson correlation coefficient between the expressions of the glutathione synthesis genes and our oxidative stress predictor. 


\section{Differential Expression Analyses}

We have used edgeR in the R package (edgeR package: https:// www.r-project.org/) to determine if a gene is differentially expressed in cancer vs. control samples of the same cancer type. $T$-test is applied to estimate the statistical significance of each gene considered as differentially expressed, using 0.05 as the cut-off.

\section{Linear Regression Analysis}

We have conducted a linear regression analysis of the observed mutation rates, $\boldsymbol{Y}_{n}$, in n samples of a specified cancer type against the expressions of $\mathrm{m}$ selected genes across $\mathrm{n}$ samples, $\boldsymbol{X}_{\boldsymbol{n}, \boldsymbol{m}}$, so that residual $\|\varepsilon\|$ is as small as possible as defined below:

$$
Y=X B+\varepsilon
$$

where $\boldsymbol{B}_{\boldsymbol{m}}$ is a coefficient vector with its $\mathrm{m}$ values to be determined through solving this optimization problem. To avoid using too many genes in the regression analysis, we have included a penalty for penalizing using more variables than necessary.

$$
Y=X B+\varepsilon+\lambda_{m}
$$

where $\lambda$ is an (adjustable) constant. This problem can be solved using a least squared regression analyses in the following form:

$$
\operatorname{argmin}_{B \epsilon R^{m}}\left(Y-X B-\lambda_{m}\right)^{2}
$$

For each $\boldsymbol{Y}$, we have retrieved the number of point mutations per cancer genome. Then for the $\mathrm{n}$ cancer genomes of each of the 14 cancer types, we have n numbers, namely the mutation number divided by the number of genes $(20,000)$, which gives rise to the $\boldsymbol{Y}$. For the ith row of $\boldsymbol{X}$, we have retrieved the expression data of $m$ selected genes in the ith sample of the same cancer type. For each regression analysis, we have used the $\mathrm{R}$ package to solve the minimization problem. The regression result has a $p$ value associated with each of the $\mathrm{m}$ values of $\boldsymbol{B}$. We then remove those genes with insignificant $p$-values, i.e., $>0.05$, for the second round of regression analyses mentioned in the Results section.

\section{Correlation Analyses}

We have used Pearson correlation coefficient to calculate the linear correlation between two lists of numbers, with one being the combined gene-expression data and the other being mutation rates of the same samples.

\section{Pathway Enrichment Analysis}

We have conducted a pathway enrichment analysis over a given set of genes found to be strongly correlated with our predictor for oxidative stress using DAVID tool (David

\section{REFERENCES}

Alfaradhi, M. Z., Fernandez-Twinn, D. S., Martin-Gronert, M. S., Musial, B., Fowden, A., and Ozanne, S. E. (2014). Oxidative stress and altered lipid homeostasis in the programming of offspring fatty liver by maternal links: https://david.ncifcrf.gov/) against the combined database of GO/Biological Process, KEGG and Reactome pathways. A pathway is considered enriched if tits adjusted $p<0.05$.

\section{Assessment of the Level of Contribution by Each Enzyme Subclass in Regression Model}

For each derived regression model against selected enzymes, the following is used to estimate the level of contribution by each subclass of enzymes. The package used for linear repression construction provides a $p$-value for each selected gene, indicating the level of the gene's contribution to the regression result with smaller $p$-value representing higher level of contribution. We then use the product of the $p$-values of the selected genes from the same enzyme subclass as the $p$-value of the subclass.

\section{SOFTWARE AVAILABILITY}

Our predictor is written in $\mathrm{R}$ language and is available upon request.

\section{DATA AVAILABILITY STATEMENT}

BLCA, BRCA, COAD, ESCA, HNSC, KICH, KIRC, KIRP, LIHC, LUAD, LUSC, PRAD, STAD, and THCA were downloaded from the TCGA data portal (TCGA data portal: https://portal.gdc. cancer.gov/). Human enzyme information, including gene names and the relevant enzyme classes, is downloaded from BRENDA (BRENDA links: https://www.brenda-enzymes.org/).

\section{AUTHOR CONTRIBUTIONS}

LL designed the method, carried out computational analyses, analyzed computational results, and wrote the article. HC supported in data analysis. YX conceived the project, reviewed computational analyses, and revised the article.

\section{ACKNOWLEDGMENTS}

LL wants to extend her thanks to members of the Computational Systems Biology Lab (CSBL) in the University of Georgia, for their helpful discussions related to the study here.

\section{SUPPLEMENTARY MATERIAL}

The Supplementary Material for this article can be found online at: https://www.frontiersin.org/articles/10.3389/fgene. 2020.00494/full\#supplementary-material 
breaks. Proc Natl Acad Sci USA. 107, 2207-2212. doi: 10.1073/pnas.09088 67107

Bellezza, I., Mierla, A. L., and Minelli, A. (2010). Nrf2 and NF-kB and their concerted modulation in cancer pathogenesis and progression. Cancers 2, 483-497. doi: 10.3390/cancers2020483

Campbell, K., Vowinckel, J., Keller, M. A., and Ralser, M. (2016). Methionine metabolism alters oxidative stress resistance via the pentose phosphate pathway. Antioxid. Redox Signal. 24, 543-547. doi: 10.1089/ars.2015.6516

Cerutti, P. A., and Trump, B. F. (1993). Inflammation and oxidative stress in carcinogenesis. Cancer Cells 3, 1-7. doi: 10.1007/978-1-4615-3520-1_75

Chan, H. P., Lewis, C., and Thomas, P. S. (2010). Oxidative stress and exhaled breath analysis: a promising tool for detection of lung cancer. Cancers 2, 32-42. doi: $10.3390 /$ cancers 2010032

Chang, D., Wang, F., Zhao, Y. S., and Pan, H. Z. (2008). Evaluation of oxidative stress in colorectal cancer patients. Biomed. Environ. Sci. 21, 286-289. doi: 10.1016/S0895-3988(08)60043-4

Chung, Y. J., Robert, C., Gough, S. M., Rassool, F. V., and Aplan, P. D. (2014). Oxidative stress leads to increased mutation frequency in a murine model of myelodysplastic syndrome. Leuk. Res. 38, 95-102. doi: 10.1016/j.leukres.2013.07.008

Conti, A., Guli, C., La Torre, D., Tomasello, C., Angileri F. F., and M'Hammed, A. (2010). Role of inflammation and oxidative stress mediators in gliomas. Cancers 2, 693-712. doi: 10.3390/cancers2020693

Dalle-Donne, I., Rossi, R., Giustarini, D., Milzani, A., and Colombo, R. (2003). Protein carbonyl groups as biomarkers of oxidative stress. Clin. Chim. Acta 329, 23-38. doi: 10.1016/S0009-8981(03)00003-2

Dayem, A. A., Choi, H. Y., Kim, J. H., and Cho, S. G. (2010). Role of oxidative stress in stem, cancer, and cancer stem cells. Cancers 2, 859-884. doi: $10.3390 /$ cancers 2020859

Dewald, J. H., Colomb, F., Bobowski-Gerard, M., Groux-Degroote, S., and Delannoy, P. (2016). Role of cytokine-induced glycosylation changes in regulating cell interactions and cell signaling in inflammatory diseases and cancer. Cells 5:43. doi: 10.3390/cells5040043

Doudican, N. A., Song, B., Shadel, G. S., and Doetsch, P. W. (2005). Oxidative DNA damage causes mitochondrial genomic instability in Saccharomyces cerevisiae. Mol. Cell. Biol. 25, 5196-5204. doi: 10.1128/MCB.25.12.5196-5204.2005

Farah, I. O. (2005). Assessment of cellular responses to oxidative stress using MCF7 breast cancer cells. black seed (N. Sativa L.) extracts and H2O2. Int. J. Environ. Res. Public Health. 2, 411-419. doi: 10.3390/ijerph2005030005

Fernando, N., Wickremesinghe, S., Niloofa, R., Rodrigo, C., Karunanayake, L., de Silva, H. J., et al. (2016). Protein carbonyl as a biomarker of oxidative stress in severe leptospirosis, and its usefulness in differentiating leptospirosis from dengue infections. PLoS ONE 11:e0156085. doi: 10.1371/journal.pone.0156085

Fionda, C., Abruzzese, M. P., Santoni, A., and Cippitelli, M. (2016). Immunoregulatory and effector activities of nitric oxide and reactive nitrogen species in cancer. Curr. Med. Chem. 23, 2618-2636. doi: 10.2174/0929867323666160727105101

Fitzgerald, D. M., Hastings, P. J., and Rosenberg, S. M., (2017). Stress-induced mutagenesis: implications in cancer and drug resistance. Ann. Rev. Cancer Biol. 1, 119-140. doi: 10.1146/annurev-cancerbio-050216-121919

Freitas, H. R., Ferreira, G. D. C., Trevenzoli, I. H., Oliveira, K. J., and de Melo Reis, R. A. (2017). Fatty acids, antioxidants and physical activity in brain aging. Nutrients 9:1263. doi: 10.3390/nu9111263

Gibellini, L., Pinti, M., Nasi, M., De Biasi, S., Roat, E., Bertoncelli, L., et al. (2010). Interfering with ROS metabolism in cancer cells: the potential role of quercetin. Cancers 2, 1288-1311. doi: 10.3390/cancers2021288

Giustarini, D., Dalle-Donne, I., Lorenzini, S., Selvi, E., Colombo, G., Milzani, A., et al. (2012). Protein thiolation index (PTI) as a biomarker of oxidative stress. Free Radic. Biol. Med. 53, 907-915. doi: 10.1016/j.freeradbiomed.2012.06.022

Giustarini, D., Galvagni, F., Colombo, G., Dalle-Donne, I., Milzani, A., Aloisi, A. M., et al. (2017). Determination of protein thiolation index (PTI) as a biomarker of oxidative stress in human serum. Anal. Biochem. 538, 38-41. doi: 10.1016/j.ab.2017.09.010

Gorrini, C., Harris, I. S., and Mak, T. W. (2013). Modulation of oxidative stress as an anticancer strategy. Nat. Rev. Drug Discov. 12, 931-947. doi: $10.1038 / \mathrm{nrd} 4002$

Graham, S. H. (2016). Modification of ubiquitin C-terminal hydrolase L1 by reactive lipid species: role in neural regeneration and diseases of aging. Neural Regen. Res. 11, 908-909. doi: 10.4103/1673-5374.184482
Hacişevki, A., Baba, B., Gonenc, A., and Aslan, S. (2012). Protein carbonyl content as the most general and well-used biomarker of severe oxidative stress. Oxid. Commun. 35, 413-422. Available online at: https://www.researchgate.net/ publication/281595280_Protein_carbonyl_content_as_the_most_general_ and_well-used_biomarker_of_severe_oxidative_stress

Halder, S. R., and Bhattacharyya, M. (2014). Oxidative stress: lipid peroxidation products as predictors in disease progression. J. Exp. Integr. Med. 4, 151-164. doi: 10.5455/jeim.210414.rw.007

Heneberg, P. (2019). Redox regulation of hexokinases. Antioxid. Redox Signal. 30, 415-442. doi: 10.1089/ars.2017.7255

Henkler, F., Brinkmann, J., and Luch, A. (2010). The role of oxidative stress in carcinogenesis induced by metals and xenobiotics. Cancers 2, 376-396. doi: $10.3390 /$ cancers 2020376

Higdon, A., Diers, A. R., Oh, J. Y., Landar, A., and Darley-Usmar, V. M. (2012). Cell signalling by reactive lipid species: new concepts and molecular mechanisms. Biochem. J. 442, 453-464. doi: 10.1042/BJ20111752

Itabe, H. (2012). Oxidized low-density lipoprotein as a biomarker of in vivo oxidative stress: from atherosclerosis to periodontitis. J. Clin. Biochem. Nutr. 51, 1-8. doi: 10.3164/jcbn.11-00020R1

Karlenius, T. C., and Tonissen, K. F. (2010). Thioredoxin and cancer: a role for thioredoxin in all states of tumor oxygenation. Cancers 2, 209-232. doi: $10.3390 /$ cancers 2020209

Kato, S., Karino, K., Hasegawa, S., Nagasawa, J., Nagasaki, A., Eguchi, M., et al. (1995). Octacosanol affects lipid metabolism in rats fed on a high-fat diet. $\mathrm{Br}$. J. Nutr. 73, 433-441. doi: 10.1079/BJN19950045

Kong, X., Wang, G., and Li, S. (2007). Antioxidation and ATPase activity in the gill of mud crabScylla serrataunder cold stress. Chin. J. Oceanol. Limnol. 25, 221-226. doi: 10.1007/s00343-007-0221-7

Kruk, J., and Aboul-Enein, H. Y. (2017). Reactive oxygen and nitrogen species in carcinogenesis: implications of oxidative stress on the progression and development of severalcancer types. Mini Rev. Med. Chem. 17, 904-919. doi: $10.2174 / 1389557517666170228115324$

Leone, A., Roca, M. S., Ciardiello, C., Costantini, S., and Budillon, A. (2017). Oxidative stress gene expression profile correlates with cancer patient poor prognosis: identification of crucial pathways might select novel therapeutic approaches. Oxid. Med. Cell. Longev. 2017, 1-18. doi: 10.1155/2017/2597581

Limoli, C. L., and Giedzinski, E. (2003). Induction of chromosomal instability by chronic oxidative stress. Neoplasia 5, 339-346. doi: 10.1016/S1476-5586(03)80027-1

Limón-Pacheco, J., and Gonsebatt, M. E. (2009). The role of antioxidants and antioxidant-related enzymes in protective responses to environmentally induced oxidative stress. Mutat. Res. Genet. Toxicol. Environ. Mutagen. 674, 137-147. doi: 10.1016/j.mrgentox.2008.09.015

Liu, L. P., Li, B., Shuai, T. K., Zhu, L., and Li, Y.-M. (2018). Deletion of soluble epoxide hydrolase attenuates mice Hyperoxic acute lung injury. $B M C$ Anesthesiol. 18,1-7. doi: 10.1186/s12871-018-0490-z

Mantovani, G., Macciò, A., Madeddu, C., Mura, L., Gramignano, G., Lusso, M. R., et al. (2010). Quantitative evaluation of oxidative stress, chronic inflammatory indices and leptin in cancer patients: correlation with stage and performance status. Int. J. Cancer 98, 84-91. doi: 10.1002/ijc.10143

Markkanen, E. (2017). Not breathing is not an option: how to deal with oxidative DNA damage. DNA Repair 59, 82-105. doi: 10.1016/j.dnarep.2017.09.007

Martínez, Y., Li, X., Liu, G., Bin, P., Yan, W., Más, D., et al. (2017). The role of methionine on metabolism, oxidative stress, and diseases. Amino Acids 49, 1-8. doi: $10.1007 / \mathrm{s} 00726-017-2494-2$

Massi, P., Valenti, M., Solinas, M., and Parolaro, D. (2010). Molecular mechanisms involved in the antitumor activity of cannabinoids on gliomas: role for oxidative stress. Cancers 2, 1013-1026. doi: 10.3390/cancers2021013

Niki, E. (2008). Lipid peroxidation products as oxidative stress biomarkers. Biofactors 34, 171-180. doi: 10.1002/biof.5520340208

Ortega, A. L., Mena, S., and Estrela, J. M. (2010). Oxidative and nitrosative stress in the metastatic microenvironment. Cancers 2, 274-304. doi: $10.3390 /$ cancers 2020274

Osman, M. Y., Rahman, T., Ismail, T. S., Azlina, A. R., and Nawawi, H. (2016). Investigation of oxidative stress status in metabolic syndrome patients using lipid peroxidation biomarkers. Int. Arch. Med. 9, 1-10. doi: 10.3823/1879

Osmundsen, H., Cervenka, J., and Bremer, J. (1982). A role for 2,4-enoylCoA reductase in mitochondrial beta-oxidation of polyunsaturated fatty acids. Effects of treatment with clofibrate on oxidation of polyunsaturated 
acylcarnitines by isolated rat liver mitochondria. Biochem. J. 208, 749-757. doi: 10.1042/bj2080749

Pizzimenti, S., Toaldo, C., Pettazzoni, P., Dianzani, M. U., and Barrera, G. (2010). Effects of reactive oxygen species and the lipid peroxidation product 4-hydroxynonenal in the hallmarks of cancer. Cancers 2, 338-363. doi: 10.3390/cancers2020338

Rajput, I. R., Li, Y. L., Xu, X., Huang, Y., Zhi, W. C., Yu, D. Y., et al. (2013). Supplementary effects of Saccharomyces boulardii and Bacillus subtilis B10 on digestive enzyme activities, antioxidation capacity and blood homeostasis in broiler. Int. J. Agric. Biol. 15, 1560-8530. Available online at: https:// www.researchgate.net/publication/236872023_Supplementary_Effects_of_ Saccharomyces_boulardii_and_Bacillus_subtilis_B10_on_Digestive_Enzyme Activities_Antioxidation_Capacity_and_Blood_Homeostasis_in_Broiler

Rao, C. V., Asch, A. S., and Yamada, H. Y. (2017). Frequently mutated genes/pathways and genomic instability as prevention targets in liver cancer. Carcinogenesis 38, 2-11. doi: 10.1093/carcin/bgw118

Reczek, C. R., and Chandel, N. S. (2017). The two faces of reactive oxygen species in cancer. Ann. Rev. Cancer Biol. 1, 79-98. doi: 10.1146/annurev-cancerbio-041916-065808

Reuter, S., Gupta, S. C., Chaturvedi, M. M., and Aggarwal, B. B. (2010). Oxidative stress, inflammation, and cancer: how are they linked? Free Radic. Biol. Med. 49, 1603-1616. doi: 10.1016/j.freeradbiomed.2010.09.006

Richard, D., Kefi, K., Barbe, U., Bausero, P., and Visioli, F. (2008). Polyunsaturated fatty acids as antioxidants. Pharmacol. Res. 57, 451-455. doi: 10.1016/j.phrs.2008.05.002

Santos, C. R., and Schulze, A. (2012). Lipid metabolism in cancer. FEBS J. 279, 2610-2623. doi: 10.1111/j.1742-4658.2012.08644.x

Schultz, M. A., Abdel-Mageed, A. B., and Mondal, D. (2010). The Nrf1 and Nrf2 balance in oxidative stress regulation and androgen signaling in prostate cancer cells. Cancers 2, 1354-1378. doi: 10.3390/cancers2021354

Schulz, J. B., Lindenau, J., Seyfried, J., and Dichgans, J. (2000). Glutathione, oxidative stress and neurodegeneration. Eur. J. Biochem. 267, 4904-4911. doi: 10.1046/j.1432-1327.2000.01595.x

Selvaraj, N., Bobby, Z., Das, A. K., Ramesh, R., and Koner, B. C. (2002). An evaluation of level of oxidative stress and protein glycation in nondiabetic Undialyzed chronic renal failure patients. Clin. Chim. Acta 324, 45-50. doi: 10.1016/S0009-8981(02)00211-5

Sies, H. (1997). Oxidative stress: oxidants and antioxidants. Exp. Physiol. Transl. Integr. 82, 291-295. doi: 10.1113/expphysiol.1997.sp004024

Slane, B. G., Aykin-Burns, N., Smith, B. J., Kalen, A. L., Goswami, P. C., Domann, F. E., et al. (2006). Mutation of succinate dehydrogenase subunit $\mathrm{C}$ results in increased $\mathrm{O}_{2}$--oxidative stress, and genomic instability. Cancer Res. 66, 7615-7620. doi: 10.1158/0008-5472.CAN-06-0833

Soory, M. Oxidative Stress Induced Mechanisms in the Progression of Periodontal Diseases and Cancer: A Common Approach to Redox Homeostasis? Cancers (2010). 2, 670-692. doi: 10.3390/cancers2020670

Sosa, V., Moliné, T., Somoza, R., Paciucci, R., Kondoh, H., and LLeonart, M. E. (2013). Oxidative stress and cancer: an overview. Ageing Res. Rev. 12, 376-390. doi: 10.1016/j.arr.2012.10.004

Sun, W. G., Weydert, C. J., Zhang, Y., Yu, L., Liu, J., Spitz, D. R., et al. (2010). Superoxide enhances the antitumor combination of AdMnSOD plus BCNU in breast cancer. Cancers 2, 68-87. doi: 10.3390/cancers 2010068
Takeyama, K., Dabbagh, K., Jeong Shim, J., Dao-Pick, T., Ueki, I. F., and Nadel, J. A. (2000). Oxidative stress causes mucin synthesis via transactivation of epidermal growth factor receptor: role of neutrophils. J. Immunol. 164, 1546-1552. doi: 10.4049/jimmunol.164.3.1546

Tang, K., Zhao, Y., Li, H., Zhu, M., Li, W., Liu, W., et al. (2017). Translocase of inner membrane 50 functions as a novel protective regulator of pathological cardiac hypertrophy. J. Am. Heart Assoc. 6:e004346. doi: 10.1161/JAHA.116.004346

Teppner, M., Böss, F., Ernst, B., and Pähler, A. (2015). Application of lipid peroxidation products as biomarkers for flutamide-induced oxidative stress in vitro. Toxicol. Lett. 238, 53-59. doi: 10.1016/j.toxlet.2015.08.005

Tubbs, A., and Nussenzweig, A. (2017). Endogenous DNA damage as a source of genomic instability in cancer. Cell 168, 644-656. doi: 10.1016/j.cell.2017.01.002

Valle, A., Oliver, J., and Roca, P. (2010). Role of uncoupling proteins in cancer. Cancers 2, 567-591. doi: 10.3390/cancers2020567

Wangpaichitr, M., Wu, C., You, M., Maher, J. C., Dinh, V., Feun, L. G., et al. (2009). $\mathrm{N}^{\prime} 1, \mathrm{~N}^{\prime}$ 3-dimethyl-N'1, N'3-bis (phenylcarbonothioyl) propanedihydrazide (Elesclomol) selectively kills cisplatin resistant lung cancer cells through reactive oxygen species (ROS). Cancers 1, 23-38. doi: 10.3390/cancers1010023

Wieczorek, E., Jablonowski, Z., Tomasik, B., Gromadzinska, J., Jablonska, E., Konecki, T., et al. (2017). Different gene expression and activity pattern of antioxidant enzymes in bladder cancer. Anticancer Res. 37, 841-848. doi: 10.21873/anticanres.11387

Wu, Y. M., Nowack, D. D., Omenn, G. S., and Haab, B. B. (2009). Mucin glycosylation is altered by pro-inflammatory signaling in pancreatic-cancer cells. J. Proteome Res. 8, 1876-1886. doi: 10.1021/pr8008379

Xu, B., Wang, W., Guo, H., Sun, Z., Wei, Z., Zhang, X., et al. (2014). Oxidative stress preferentially induces a subtype of micronuclei and mediates the genomic instability caused by p53 dysfunction. Mutat. Res. 770, 1-8. doi: 10.1016/j.mrfmmm.2014.08.004

Zhang, F., and Du, G. (2012). Dysregulated lipid metabolism in cancer. World J. Biol. Chem. 3, 167-174. doi: 10.4331/wjbc.v3.i8.167

Zhang, H., Lei, Y., Yuan, P, Li, L., Luo, C., Gao, R., et al. (2014). ROS-mediated autophagy induced by dysregulation of lipid metabolism plays a protective role in colorectal cancer cells treated with gambogic acid. PLOS ONE 9:e96418. doi: 10.1371/journal.pone.0096418

Zhang, W., Trachootham, D., Liu, J., Chen, G., Pelicano, H., Garcia-Prieto, C., et al. (2012). Stromal control of cystine metabolism promotes cancer cell survival in chronic lymphocytic leukaemia. Nat. Cell Biol. 14, 276-286. doi: $10.1038 /$ ncb2432

Conflict of Interest: The authors declare that the research was conducted in the absence of any commercial or financial relationships that could be construed as a potential conflict of interest.

Copyright (c) $2020 \mathrm{Liu}, \mathrm{Cui}$ and Xu. This is an open-access article distributed under the terms of the Creative Commons Attribution License (CC BY). The use, distribution or reproduction in other forums is permitted, provided the original author(s) and the copyright owner(s) are credited and that the original publication in this journal is cited, in accordance with accepted academic practice. No use, distribution or reproduction is permitted which does not comply with these terms. 